\title{
Heavy Flavor Measurements at RHIC in the Near Future
}

\author{
$\mathrm{Nu} \mathrm{Xu}$ \\ Nuclear Science Division, MS 70R0319, Lawrence Berkeley National Laboratory \\ Berkeley, CA 94720, USA
}

\begin{abstract}
We discuss the recent results on open charm measurements at RHIC. The heavy flavor upgrade program for both PHENIX and STAR experiments are briefly discussed. The completion of the program will yield important information on light flavor thermalization of the partonic matter created in high-energy nuclear collisions at RHIC. A new era of RHIC is ahead of us with the progress of the upgrade program.
\end{abstract}

\section{Introduction}

Lattice QCD calculations [1,2] predict a transition or fast cross-over between QGP and the hadronic state at $T_{c} \approx 150-180 \mathrm{MeV}$ with vanishing baryon density. Including finite baryon density does not affect the general properties of the transition in Lattice QCD [3]. The energy density at the transition point is determined to be $\epsilon_{c} \approx 0.7-1.0 \mathrm{GeV} / \mathrm{fm}^{3}$. Under the same condition, chiral symmetry restoration also happens [1]. Therefore experimentally one would search for signatures of both QGP formation and in-medium effects of hadron properties.

Due to the large mass, heavy flavor (charm and bottom) production is a powerful tool for study the properties of the hot and dense medium created in high-energy nuclear collisions at RHIC. At the relatively low transverse momentum region, the collective motion of the heavy flavor will be a sensitive signal for the thermalization of light flavors at RHIC.

Recently reported charm cross sections from $\sqrt{s_{\mathrm{NN}}}=200 \mathrm{GeV}$ collisions are compared with model predictions in Fig. 1. All data are from mid-rapidity only. All data points are shifted in the figure for a better display. Circles and 
triangles are data from STAR [4,5] and PHENIX [6-8] experiments, respectively. Shaded boxes represent the systematic errors. In case of STAR measurement, the cross sections are extracted from both reconstructed D-mesons and semi-leptonic electron and muon decays while for the PHENIX experiment, all data are from electron decay measurements. Systematic errors dominate the uncertainties in all measurements although STAR data points are consistently higher than that of PHENIX's. PHENIX has much lower material budget so their electron measurements are relatively cleaner. The most recent results [8] show that the charm cross section is in some agreement with a fixedorder-plus-next-to-leading-log (FONLL) model calculation [12], especially at the electron $p_{T} \geq 1 \mathrm{GeV} / \mathrm{c}$ region.

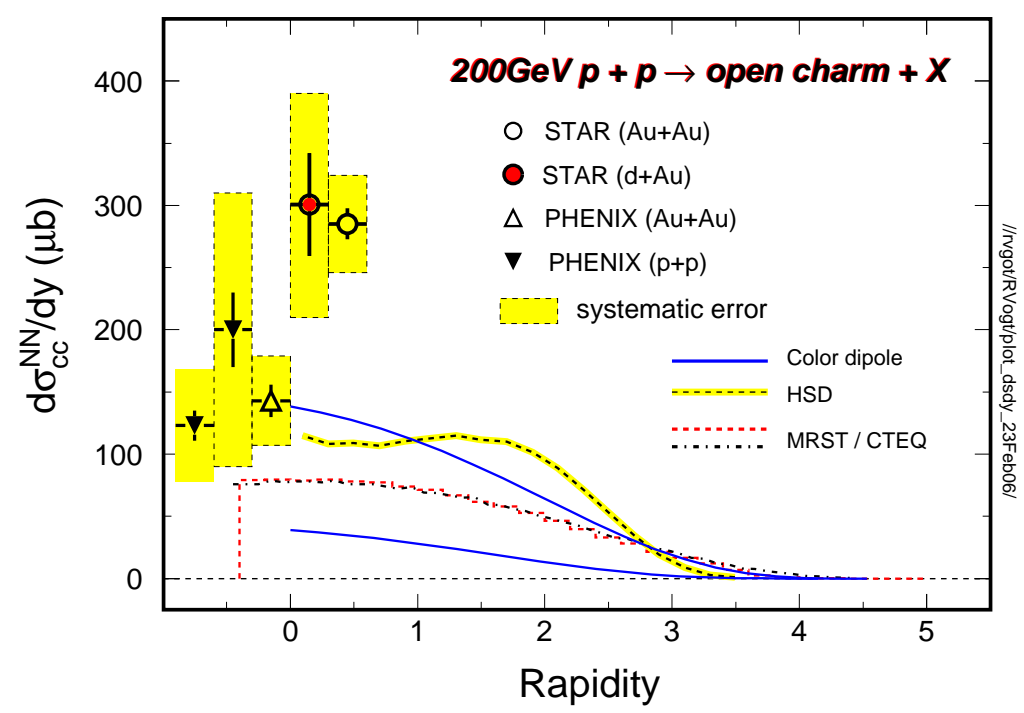

Fig. 1. Differential charm cross section, per nucleon collisions, from $\sqrt{s_{\mathrm{NN}}}=200$ collisions at RHIC. Circles and triangles are data from STAR [4,5] and PHENIX [6-8] experiments, respectively. Solid lines, dashed-line and dot-dashed-line are model predictions [9-11].

Extracting the heavy flavor production cross section from semi-leptonic decay is a serious challenge to any experiment. In addition to the great difficulties of rejecting the photonic electrons, generated by the detector materials, one has to understand the decay kinematics. As one can see in Fig. 2, the correlation between heavy flavor hadrons and the decay-electrons are broad and the higher the $p_{T}$ the broader the correlation.

In order to overcome the problems in measuring the decayed electrons from heavy flavor hadrons, further study the hot and dense medium properties (at low $p_{T}$ region) and test the pQCD predictions in high density strong coupling region (at high $p_{T}$ ), one must directly measure heavy flavor hadron distributions. At RHIC, both PHENIX and STAR experiments upgrade programs include strong heavy flavor components. 


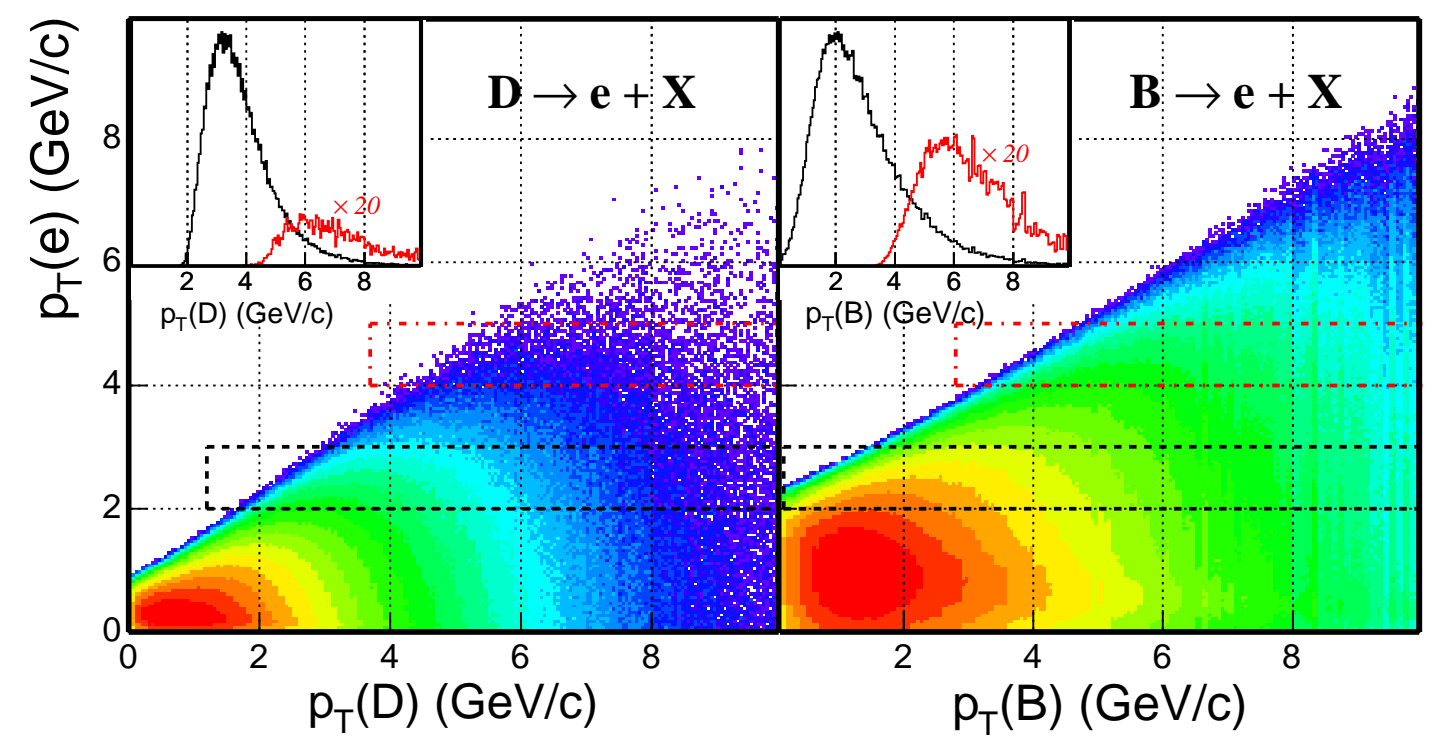

Fig. 2. Heavy flavor decayed electron transverse momenta versus open-charm (left plot) and open-bottom (right-plot) momenta. Inserted plots represent the projections to the corresponding heavy flavor distributions. The width of the electron $p_{T}$ windows are indicated in the plots by dashed-boxes.

The most exciting prospective measurement with the heavy flavor upgrades is to perform a measurement of the elliptic flow of $\mathrm{D}$ mesons down to very low $p_{T}$ values. It is generally accepted that elliptic flow is established in the partonic phase. If charm quarks, with a mass much larger than the temperature of the system, undergo elliptic flow then it has to arise from many collisions with the abundant light quarks and gluons. Thus, flow of charm quarks can be taken as a probe for frequent re-scatterings of light quarks and is an indication of thermalization that may be reached in the early stages of heavy ion collisions at RHIC. We believe that proof of thermalization constitutes the last step towards the establishment of the QGP at RHIC and this measurement requires a detector with very low material budget.

\section{RHIC heavy flavor upgrades}

To provide the accurate track reconstruction required by the intended measurements, the detector design must include: 1) small radiation length to minimize multiple scattering, 2) sensors positioned close to the interaction region, and 3) resolution sufficient to resolve the secondary decay vertexes from the primary vertex. The $c \tau$ of the D-meson are in the order of $100-300 \mu \mathrm{m}$, and the charm baryon $\Lambda_{c}$ is only $\sim 100 \mu \mathrm{m}$. This sets the resolution requirements of the detector.

The PHENIX Si-upgrade: Fig. 3 shows the schematics of the PHENIX de- 


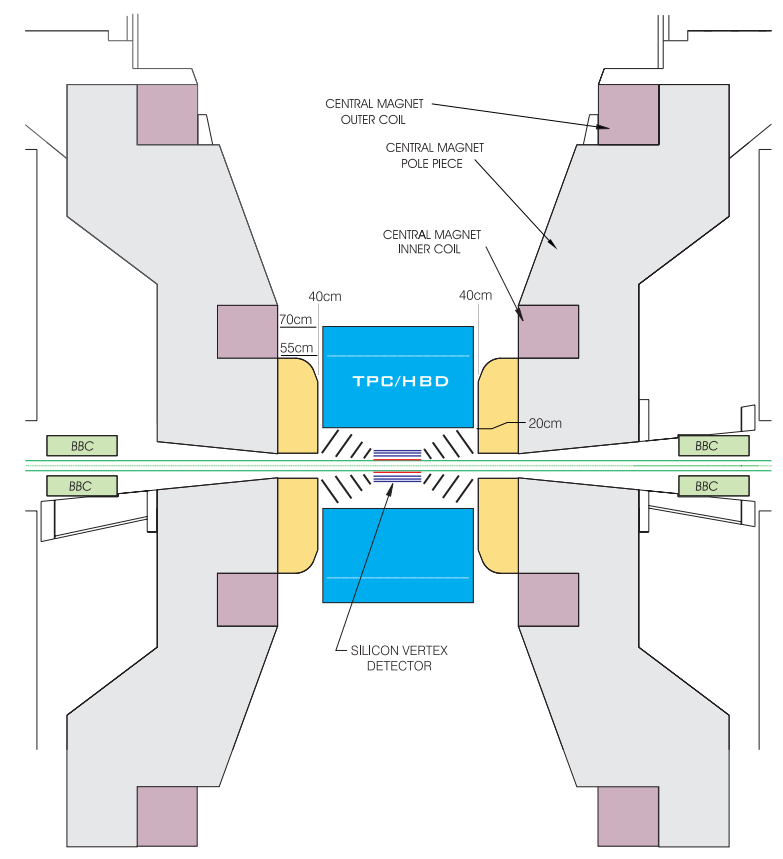

Fig. 3. Schematic view of the PHENIX detector system. The upgrade Si-barrel and Si-endcap are shown at the center of the detector.

tector system, including the upgrade components. At the center, the Si-VTX and Si-endcap-VTX are shown. The rapidity coverages are $|\eta| \leq 1.2$ and $1.2 \leq|\eta| \leq 2.7$ for the barrel VTX and endcap VTX, respectively. Combined with a hadron-blind-detector (HBD), the new system greatly enhances the displaced vertex and jets measurements [13] in full azimuth. The Si-barrel contains 2 layers of Si-hybrid pixels and 2 additional layer of Si-strip detectors. The radius of the most inner layer is about $2.5 \mathrm{~cm}$. This is determined by the size of the RHIC beam pipe and common for both PHENIX and STAR. In the central rapidity region, the charm will be measured by decayed electrons for $p_{T} \leq 2 \mathrm{GeV} / \mathrm{c}$ and by directly reconstructed D-mesons in $2 \leq p_{T} \leq 6 \mathrm{GeV} / \mathrm{c}$, and the bottom will be detected via the decay $B \rightarrow J / \psi+X$.

The STAR Si-upgrade: The STAR Heavy Flavor Tracker[15] (HFT) will use active pixel sensor technology (APS) for the detector elements. These CMOS chips can be thinned to $50 \mu \mathrm{m}$; together with the support structure, the detector layer is only $0.28 \%$ in radiation length. This minimizes the incidence of multiple scattering, and therefore the impact on the momentum resolution of the reconstructed tracks. This is critical for high precision invariant mass reconstruction. The HFT contains two layers that cover the rapidity region $|\eta| \leq 1$. The inner layer has a radius of $2.5 \mathrm{~cm}$.

The CMOS chips, developed at iReS [16], have pixels of $30 \mu \mathrm{m}$, resulting in an $8 \mu \mathrm{m}$ single-hit resolution detector. The current prototype chips have a $4 \mathrm{~ms}$ integration time; since it will be continuously read out, however, dead time will not be an issue. This does imply that there will be several events in the accep- 


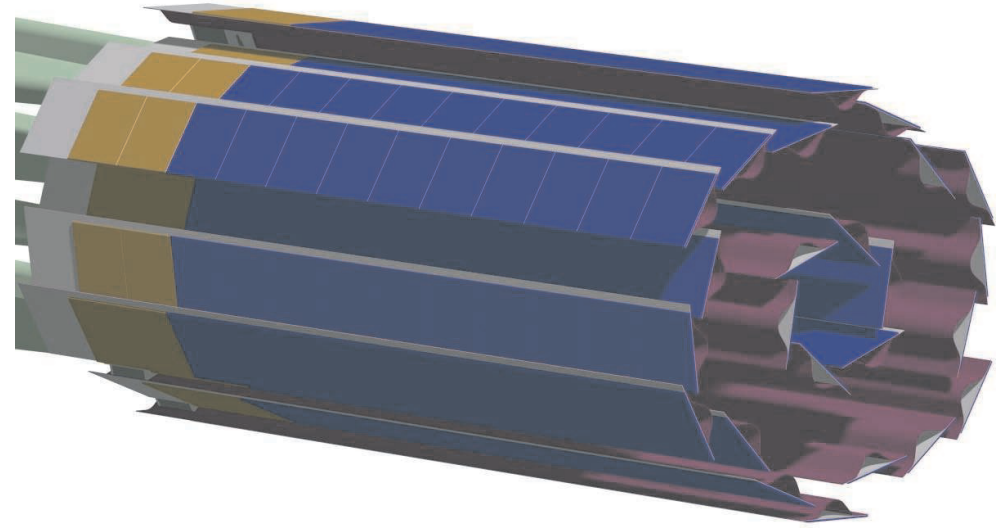

Fig. 4. Schematic view of the STAR HFT detector ladder arrangement with 6 ladders forming the inner layer and 18 ladders forming the outer layer. The width of the ladders (the dimension seen in this view) is $19 \mathrm{~mm}$. The shingled layers provide cross calibration of the ladders and provide an open geometry for ease of installation.

tance of the HFT in addition to the triggered event. These background hits, called pileup hits, are a source of ghosting and inefficiency in reconstruction $[18]$.

Compared with the PHENIX Si-VTX, the STAR HFT is relatively thinner in radiation length. It has a full azimuthal coverage that allows to reconstruct charm hadrons with $p_{T} \geq 0.5 \mathrm{GeV}$. Combined with the large acceptance TPC, the STAR HFT will bebable to measure open charm elliptic flow $v_{2}$, the nuclear modification factor $R_{A A}$ and the correlation measurements for D-mesons[17]. The reconstruction efficiencies of open charm hadrons ranging from few percent upto few tenth of percent from $p_{T} \sim 0.5$ to $p_{T} \sim 6 \mathrm{GeV} / \mathrm{c}$.

In order to test the thermodynamic behavior of the D-meson, the most important region is $p_{T} \leq 3 \mathrm{GeV} / \mathrm{c}$ because at higher $p_{T}$ region other dynamical effects will become important (e.g. jet correlations). The proposed STAR HFT detector will be able to make precise $v_{2}$ measurements in the transverse momentum region $0.7 \leq p_{T} \leq 3 \mathrm{GeV} / \mathrm{c}$. As we have done with the hadrons from the light-flavor sector, the combined analysis of the D-meson spectra, $v_{2}$ distributions [14] and ratios will allow us to determine the charm quark collectivity and the thermodynamic nature of the medium created in heavy ion collisions at RHIC.

\section{Summary}

As shown in Fig. 5, both PHENIX and STAR heavy flavor upgrades will be ready for data between 2009 - 2011. With the upgrades, the physics of heavy flavor collectivity and light flavor thermalization will be studied. In addition, 


\begin{tabular}{|c|c|c|c|c|c|c|c|c|c|c|}
\hline & 2002 & 2003 & 2004 & 2005 & 2006 & 2007 & 2008 & 2009 & 2010 & 2011 \\
\hline Barrel VTX & & & & & & & & 10 & & \\
\hline Endcap VTX & & & & & & & & $\mathbb{2}$ & 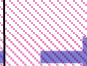 & (2) \\
\hline NCC & & & & & & & ? & 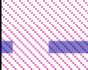 & 10 & \\
\hline MRPC TOF & $=$ & & & & & & 10 & & & \\
\hline HFT & & & & & & & - & & (b) & \\
\hline
\end{tabular}

\begin{tabular}{|cl|}
\hline & R\&D phase \\
\hline ○) & Construction phase \\
solid: & Apprody for data \\
\hline
\end{tabular}

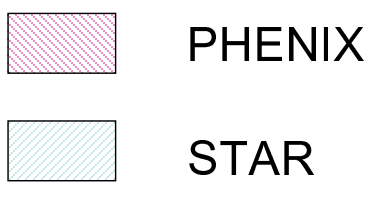

Fig. 5. Proposed schedule of the heavy flavor upgrade for both PHENIX and STAR experiments.

these upgrades provide opportunities to study the PQCD in multiparticle environment created in high-energy nuclear collisions. Whence the charm cross sections are fixed by the precision measurements of the reconstructed D-mesons, the exact cross section for bottom production can be extracted via the measured electron distributions. Note that at the similar period, LHC will deliver its first data from collisions at $\sqrt{s_{\mathrm{NN}}}=5400 \mathrm{GeV}$. A new era of deeper understanding of the partonic bulk properties will start.

The heavy flavor upgrade detectors will also offer the possibility of measuring $e^{+}$and $e^{-}$pairs down to the vector meson mass region by effectively removing the photon conversion background. Vector mesons reconstructed in the dilepton channel may have decayed at any stage of the systems evolution, since leptons are very unlikely to re-interact. Thus, studying the low mass $e^{+} e^{-}$spectrum may yield crucial insights into the nature of the system before it reaches the low-density hadronic freeze-out stage. Measuring the low-mass vector mesons through their leptonic decay channel may also yield information about the possible of chiral symmetry restoration. 


\section{Acknowledgements}

We thank the conference organizers. We are grateful for many enlightening discussions with Drs. A. Drees, X. Dong, S. Esumi, H.G. Ritter, A. Rose, K. Schweda, R. Seto, P. Sorensen, J. Thomas, Z. Xu and Y.F. Zhang. This work has been supported by the U.S. Department of Energy under Contract No. DE-AC03-76SF00098.

\section{References}

[1] H. Satz, Nucl. Phys. A715, 3c(2003).

[2] F. Karsch, Nucl. Phys. A698, 199c(2002).

[3] Z. Fodor, Nucl. Phys. A715, 319c(2003) and references there in; Z. Fodor and S. D. Katz, Phys. Lett. B534, 87(2002).

[4] J. Adams et al. (STAR Collaboration), Phys. Rev. Lett. 94, 062301(2005) [nuclex/0407006].

[5] Yifei Zhang (STAR Collaboration), proceedings of International Conference on Strangeness in Quark Matter, Los Angeles, California, 26 - 31 March 2006 [nuclex/0607011].

[6] S.S Adler et al. (PHENIX Collaboration), Phys. Rev. Lett. 96, 032001(2006) [hep-ex/0508034].

[7] S.S Adler et al. (PHENIX Collaboration), Phys. Rev. Lett. 96, 032301(2006) [nucl-ex/0510047].

[8] A. Adare, et al. (PHENIX Collaboration), hep-ex/0609010.

[9] J. Raufeisen and J. C. Peng, Phys. Rev. D67, 054008(2003) [hep-ph/0211422].

[10] R. Vogt, Int. J. Mod. Phys. E12, 211(2003) [hep-ph/0203151].

[11] E. L. Bratkovskaya, W. Cassing, H. Stoecker, and N. Xu, Phys. Rev. C71, 044901(2005) and references therein.

[12] M. Cacciari et al., Phys. Rev. Lett. 95, 122001(2005).

[13] M. Gyulassy, I. Vitev, X.-N. Wang, and B. Zhang, nucl-th/0302077 and references therein.

[14] J. Adams et al. (STAR Collaboration), Nucl. Phys. A757, 102(2005).

[15] Z. Xu et al., LBNL-PUB-5509, 2006.

[16] http://ireswww.in2p3.fr/ires/recherche/capteurs/ 
[17] X. Zhu et al., hep-ph/0604178.

[18] A. Rose et al. (STAR Collaboration), proceedings of International Conference on Strangeness in Quark Matter, Los Angeles, California, 26 - 31 March 2006. 